\title{
Late and Chronic Antibody-Mediated Rejection: Main Barrier to Long Term Graft Survival
}

\author{
Qiquan Sun ${ }^{1}$ and Yang Yang ${ }^{2}$ \\ ${ }^{1}$ Department of Renal Transplantation, The Third Affiliated Hospital, Sun Yat-Sen University, 600 Tianhe Road, \\ Guangzhou 510630, China \\ ${ }^{2}$ Department of Liver Transplantation, The Third Affiliated Hospital, Sun Yat-Sen University, 600 Tianhe Road, \\ Guangzhou 510630, China
}

Correspondence should be addressed to Qiquan Sun; sunqiquan@hotmail.com and Yang Yang; yysysu@163.com

Received 27 June 2013; Accepted 3 September 2013

Academic Editor: Xian Li

Copyright (C) 2013 Q. Sun and Y. Yang. This is an open access article distributed under the Creative Commons Attribution License, which permits unrestricted use, distribution, and reproduction in any medium, provided the original work is properly cited.

Antibody-mediated rejection (AMR) is an important cause of graft loss after organ transplantation. It is caused by anti-donorspecific antibodies especially anti-HLA antibodies. C4d had been regarded as a diagnosis marker for AMR. Although most early AMR episodes can be successfully controlled or reversed, late and chronic AMR remains the leading cause of late graft loss. The strategies which work in early AMR have limited effect on late/chronic episodes. Here, we reviewed the lines of evidence that late/chronic AMR is the leading cause of late graft loss, characteristics of late AMR, and current strategies in managing late/chronic AMR. More effort should be put on the management of late/chronic AMR to make a better long term graft survival.

\section{Introduction}

Organ transplantation now represents the treatment of choice for patients developing end stage organ failure [1]. However, despite the now routine nature of clinical transplantation, even well-matched transplants are recognized and eventually destroyed by the host immune system [2]. The emerging of a new immunosuppressant has decreased the incidence of early graft loss, and even T-cell-mediated rejection occurs; it is usually easily controlled. However, the long term graft survival remains to be improved [3]. Although it was formerly held that alloreactive $\mathrm{T}$ cells are solely responsible for graft injury, it is now well recognized that antidonor alloantibodies are also an important barrier to long term graft survival $[4,5]$. More and more lines of evidence suggest that antibodymediated rejection (AMR) is predominance cause of late term graft loss $[6,7]$, especially late occurring AMR and chronic AMR (CAMR). Thus, strategies targeting alloantibody reactivity will be helpful in prolonging long term graft survival.

\section{Antibody-Mediated Rejection}

AMR is caused by anti-donor-specific antibodies, mostly anti-HLA antibodies $[8,9]$. Some non-HLA antibodies also have been reported to induce AMR in rare cases. The phenotype of AMR ranges from hyperacute rejection, acute AMR, and chronic AMR. The diagnosis of AMR depends on typical histological lesions, C4d staining, and serum DSA detection. C4d, a protein from the classical complement activation cascade that remains attached to the site of complement activation, is regarded as a diagnosis marker for AMR. The introduction of C4d as marker of AMR aroused an everincreasing interest in recognizing mechanisms of allograft rejection. However, C4d has several limitations in the diagnosis of AMR. For instance, it can be found in the majority of grafts with stable function in $\mathrm{ABO}$-incompatible transplantations. On the other hand, a group of C4d-negative AMR has been recognized based on endothelial gene expression $[10,11]$. About $40 \%$ of patients with endothelial-associated transcripts expression and chronic AMR features demonstrated no C4d 
TABLE 1: Early versus late AMR in renal transplant recipients.

\begin{tabular}{|c|c|c|}
\hline & Early AMR & Late AMR \\
\hline Main risk factor & $\begin{array}{l}\text { Positive panel reactivity antibody before } \\
\text { transplantation, including factors causing sensitization }\end{array}$ & $\begin{array}{l}\text { Withdrawal or reduction of immunosuppressants } \\
\text { Noncompliance with immunosuppressive therapy, young } \\
\text { age }\end{array}$ \\
\hline Antibody & Mostly pre-existing donor-specific antibodies & $\begin{array}{l}\text { Mostly de novo donor-specific antibodies, especially HLA } \\
\text { class-II antibodies }\end{array}$ \\
\hline Clinical features & $\begin{array}{l}\text { Very rapid graft dysfunction, significantly decreased } \\
\text { urine output, and rapid graft dysfunction }\end{array}$ & $\begin{array}{l}\text { Proteinuria, hypertension, progressive functional } \\
\text { deterioration, and overt graft failure }\end{array}$ \\
\hline Histology & $\begin{array}{l}\text { ATN-like minimal inflammation; capillary and or } \\
\text { glomerular inflammation and/or thrombosis; } \\
\text { arterial-v3 }\end{array}$ & $\begin{array}{l}\text { May have chronic tissue injury, such as glomerular double } \\
\text { contours, peritubular capillary basement membrane } \\
\text { multilayering, interstitial fibrosis/tubular atrophy, and/or } \\
\text { fibrous intimal thickening in arteries }\end{array}$ \\
\hline Outcome & Good, mostly reversible & Usually poor \\
\hline
\end{tabular}

staining. Similarly, C4d staining is only positive in about half of patients with transplant glomerulopathy [12, 13], which is a special form of chronic AMR. C4d-positive and -negative AMR share similar degrees of glomerulitis and peritubular capillaritis, similar frequencies of concurrent cellmediated rejection, and both may occur early or late after transplantation, thus needing to be treated equally [14].

Obviously, a new marker for AMR is extremely necessary. It is reported that microcirculating inflammation is strongly correlated with alloantibody reactivity; however, whether it is can be used as maker of AMR is still of contradictory [15]. T-box expressed in T cells (T-bet), transcription factor for Th1, has been reported to be correlated with microcirculating inflammation both in acute and chronic AMR [16, 17], and the predominance of T-bet over GATA3 (transcription factor for Th2) is strongly correlated with AMR [16]. However, whether the ratio of T-bet/GATA3 can be used as a diagnosis maker for AMR needs further investigation.

2.1. Late/Chronic AMR. The importance of CAMR is increasingly recognized. It has been known as a major cause of late graft dysfunction in renal transplantation. Banff 07 consensus conference [18] described that the characteristics of chronic AMR were C4d deposition in the capillary basement membrane, the presence of circulating anti-donor antibodies, and morphologic evidence of chronic tissue injury such as glomerular double contours compatible with transplant glomerulopathy, peritubular capillary basement membrane multilayering, interstitial fibrosis/tubular atrophy, and fibrous arterial intimal thickening. Late occurring AMR may manifest as CAMR; however, according to Banff 07 meeting, the term "chronic" is not related to a certain time after transplantation but indicates morphological changes of remodeling seen in the allograft due to antibody-mediated injury [18], for example, double contours of glomerular basement membranes. Thus, it is not strange that late AMR can be acute AMR. However, both CAMR and late AMR have poor response to regular anti-AMR treatment, and they are sometimes discussed together.

2.2. Late AMR, a Special Clinical Entity? AMR episodes occurring at different time periods seem to be different clinical subentities [19-21]. They have different risk factors, different clinical manifestations, and different outcomes (Table 1). Early AMR are usually correlated with sensitization, pre-existing alloantibodies, and rapid graft dysfunction and are usually easy to be controlled; while late AMR mostly correlated with withdrawal or reduction of immunosuppressants, noncompliance with immunosuppressive therapy. There is a relatively slow but progressive graft dysfunction; some patients have anemia and hypoalbuminemia. Late AMR have little response to antirejection strategies and thus correlate with poor graft outcomes $[19,20]$. The significantly poorer outcome of late AMR is also observed in simultaneous pancreas-kidney transplantation [22], even under combined treatment of steroids, intravenous immunoglobuin (IVIG), and rituximab.

2.3. Late/Chronic AMR, Main Cause to Late Graft Loss. A eliminated term, chronic allograft nephropathy, had been regarded as a main cause of late graft loss [23]. However, chronic allograft nephropathy is actually like a can, which includs both immunological and nonimmunological parameters caused graft damage; thus, this term has been eliminated in Banff 2005 meeting [24]. Recent studies revealed that AMR is the leading cause of late grafts loss. In 2009, researchers from Dr. Holloran's group in Edmonton studied the phenotype of late kidney graft failure [6]; they found that patients with late index biopsies ( $>1$ year) frequently displayed donorspecific HLA antibody (particularly class II) and microcirculation changes, including glomerulitis, glomerulopathy, capillaritis, capillary multilayering, and C4d staining. T-cellmediated rejection rarely leads to graft failure. However, they found that $63 \%$ of late kidney failures after biopsy were attributable to AMR.

Further prospective study from the same group [7] investigated kidney transplants that progressed to failure after a biopsy for clinical indications. Similarly, they found that graft failure was rare after T-cell-mediated rejection and acute kidney injury while was common after AMR or glomerulonephritis. The majority of graft loss had evidence of AMR by the time of failure. Interestingly, pure T-cell-mediated rejection, acute kidney injury, and drug toxicity were not causes of loss. These findings are interesting and, however, 
are not strange as they are consistant with an early study which reported that all chronic rejection failures of kidney transplants preceded by development of HLA antibodies [25]. Recent study from Terasaki Foundation Laboratory revealed that $11 \%$ of the patients without detectable DSA at transplantation will have detectable DSA at 1 year; and over the next 4 years, the incidence of de novo DSA will increase to $20 \%$. After de novo DSA development, $24 \%$ of the patients will fail within 3 years [26]. Given these findings, de novo DSA, especially anti-HLA class II antibodies [27], have to be considered as a primary risk factor for late allograft loss.

2.4. Natural History of Chronic AMR. The development of chronic AMR, especially TG usually takes years, and there still lacks of a perfect animal model to mimic the lesions during chronic AMR; thus, the natural history of chronic AMR is still not clear. Recently Wiebe et al. [28] have monitored a group of renal allograft recipients with de novo DSA, they proposed that posttransplant de novo DSA is probably preceded by an antibody-free period. Then, inflammatory events such as cellular rejection or graft infection might upregulate HLA expression on endothelial cells and stimulate B-cell allorecognition and subsequent long-lived plasma cells producing de novo DSA. De novo DSA binding vascular endothelium could induce injury through the activation of complement or recruitment of neutrophils, macrophage, or natural killer cells. Sustained microvascular inflammation eventually leads to progressive tissue damage and graft dysfunction. Cellular inflammation is often concomitant of AMR $[29,30]$ in either its acute or chronic form. However, the pathogenesis remains to be determined.

2.5. Antibody-Mediated Vascular Rejection. A recent population based study [31] revealed a new type of kidney rejection not presently included in classifications, which is an antibody-mediated vascular rejection. This study included 302 cases of acute biopsy-proven rejection. Four distinct patterns of kidney allograft rejection were identified, including T-cell-mediated vascular rejection (26 patients $(9 \%)$ ), antibodymediated vascular rejection $(64(21 \%))$, T-cell-mediated rejection without vasculitis (139 (46\%)), and AMR without vasculitis $(73(24 \%))$. The graft survival is very poor in antibody-mediated vascular rejection, which has a 9 times higher graft loss incidence compared with T-cell-mediated rejection without vasculitis. Unfortunately, the authors did not provide how many of the AMR episodes were late AMR.

\section{Management of Late/Chronic AMR}

3.1. An Ounce of Prevention Is Worth a Pound of Cure. Chronic AMR is a B-cell-mediated production of immunoglobulin (Ig) G antibody against a transplanted organ. Based on this pathophysiologic condition, rituximab, IVIG, and bortezomib have been used as treatment for chronic AMR recently. However, till now, there is no standardized treatment for late/chronic AMR. The strategies that can effectively reverse early AMR do not work as well in late episodes; thus, an ounce of prevention is really worth a
TABLE 2: Strategies to treat AMR.

\begin{tabular}{|c|c|}
\hline Strategies & Mechanisms \\
\hline $\begin{array}{l}\text { Plasmapheresis [37], } \\
\text { immunoadsorption [38] }\end{array}$ & Removal of donor-specific antibodies \\
\hline IVIG $[39,40]$ & $\begin{array}{l}\text { Multiple mechanisms, basically } \\
\text { pleiotropic immunomodulation }\end{array}$ \\
\hline Rituximab $[41,42]$ & $\begin{array}{l}\text { Chimeric anti-CD } 20 \text { monoclonal } \\
\text { antibody, depleting B cells }\end{array}$ \\
\hline Bortezomib $[43,44]$ & $\begin{array}{l}\text { Proteasome inhibitor, may cause } \\
\text { apoptosis of normal plasma cells which in } \\
\text { turn decreases alloantibody production }\end{array}$ \\
\hline Eculizumab $[45,46]$ & $\begin{array}{l}\text { Humanized monoclonal antibody } \\
\text { anti-C5 }\end{array}$ \\
\hline $\begin{array}{l}\text { Mycophenolic acid, } \\
\text { tacrolimus }[34,35]\end{array}$ & Inhibit production of DSA \\
\hline Splenectomy $[47,48]$ & $\begin{array}{l}\text { Immediate reduction of the B-cell and } \\
\text { plasma cell pool }\end{array}$ \\
\hline
\end{tabular}

pound of cure [32]. As late AMR usually is caused by de novo DSA, posttransplant HLA alloantibody monitoring is of great importance for the prevention of antibody-mediated allograft injury [33]. Prevention of nonadherence and insufficient immunosuppression are additional important issues in the prevention of antibody-mediated allograft injury, as these factors are risk factor for late AMR. A recent study based on $\mathrm{ABO}$-incompatible renal transplantation revealed that B-cell depletion protocols, such as splenectomy or rituximab administration, could reduce chronic AMR after kidney transplantation. Finally, the triple immunosuppressants protocol including mycophenolic acid, tacrolimus, and steroid can control antidonor antibody production in renal allograft recipients with chronic rejection [34] and seem to be superior to others in treating AMR $[35,36]$; however, whether it can prevent the development of late AMR is not clear, see Table 2.

3.2. Rituximab/IVIG. Several single center studies showed that the combination treatment with rituximab/IVIG may be a useful strategy for the treatment of chronic AMR. In 2008, a German group [49] published their pilot study in six pediatric renal transplant recipients with chronic AMR. Their treatment regimen was four weekly doses of IVIG ( $1 \mathrm{~g} / \mathrm{kg}$ body weight per dose), followed by a single dose of rituximab $\left(375 \mathrm{mg} / \mathrm{m}^{2}\right.$ body surface area) 1 week after the last IVIG infusion. Four of the six patients had good response to this treatment; they had improved or stabilized eGFR. Further prospective studies from the same group showed that under this treatment, in the means of eGFR, $70 \%$ patients responded to treatment as measured 6 months after intervention, and this response persisted over a 24-month observation period. The rationale for the rituximab/IVIG treatment was to use IVIG for its immunomodulatory action and then rituximab for prevention of further antibody production.

At the year 2009, Fehr et al. [50] published his work of using rituximab/IVIG treatment on four adult patients with chronic AMR. The result showed that rituximab/IVIG treatment improved kidney allograft function in all four 
patients, and donor-specific antibodies were reduced in 2 of 4 patients. The treatment regimen of this study was that on diagnosis of chronic AMR, all patients received intravenous steroid pulses (500-1000 mg once daily for 3 to 5 days) and rituximab $\left(375 \mathrm{mg} / \mathrm{m}^{2}\right.$ once on day 1$)$, whereas IVIG $(0.4 \mathrm{~g} / \mathrm{kg}$ once daily on day 2 to 5$)$ was given only to 3 patients. About the treatment safety, 3 out of 4 patients underwent therapy with rituximab/IVIG without side effects. One patient had severe, possibly rituximab-associated lung toxicity. Their study showed that rituximab/IVIG may be a useful strategy for the treatment of chronic AMR. Another pilot study showed that rituximab/IVIG treatment took effect in 3 out of 4 patients. Early stage of chronic AMR has better response than advanced stage [51]. Anyway, although rituximab/IVIG treatment takes effects in some CAMR cases, it is far from comparable to early AMR cases [22]. A retrospective study from Massachusetts General Hospital [52] studied the effect of rituximab followed by standard maintenance immunosuppression, they found that this protocol shows a therapeutic effect in 8 out of 14 CAMR. Response to rituximab was defined as decline or stabilization of serum creatinine for at least one year in this study.

3.3. Bortezomib. Bortezomib is a proteosome inhibitor that leads in vitro to apoptosis of alloantibody-producing plasma cells [53]. It has shown promising effect in early AMR cases [43]. Early reports of bortezomib-based AMR treatment demonstrated the ability of bortezomib to deplete plasma cells producing DSA, reduce DSA levels, provide histological improvement or resolution, and improve renal allograft function [54]. Initial results from a multicenter study showed [55] that bortezomib-based regimen reversed AMR in adult kidney, kidney/pancreas, and pediatric heart transplant recipients; a common bortezomib-based regimen demonstrated substantial DSA reductions, with more than half of the patients achieving a $45.0 \%$ reduction in DSA level. However, plasmapheresis has been performed every third day immediately before bortezomib therapy. In a chronic AMR rat cardiac transplant model, administration of bortezomib 60 or 80 days after transplantation may reduce antidonor MHC classes I and II Abs. Histological improvements were also observed with bortezomib administration, including reduction in $\mathrm{C} 4 \mathrm{~d}$ expression, interstitial fibrosis, and vasculopathy [56]. Unfortunately, it is not as effective in late AMR cases. Walsh et al. treated 30 episodes of AMR, and they found that early AMR patients demonstrated greater reduction in DSA and histologic resolution/improvement. They concluded that early and late AMR exhibit distinct immunologic characteristics and respond differently to proteosome inhibitor therapy.

3.4. Eculizumab. As complement plays an important role in the pathogenesis of AMR, complement-blocking agents could be used for the treatment of AMR. Eculizumab is a humanized monoclonal antibody against complement C5. It can bind to the $\mathrm{C} 5$ protein and inhibit conversion of $\mathrm{C} 5$ to $\mathrm{C} 5 \mathrm{~b}$, thus preventing formation of the membrane attack complex (C5b-9). Eculizumab has been used to rescue atypical hemolytic uremic syndrome after renal transplantation [57]. A prospective study showed that eculizumab can reduce the incidence of AMR and transplant glomerulopathy in highly sensitized individuals when administered immediately after transplant [58]. Cases had been reported that eculizumab reverse AMR is associated with thrombotic microangiopathy [59]; it can even reverse severe AMR episode refractory to salvage splenectomy and daily plasmapheresis in $\mathrm{ABO}$ incompatible (ABOI) living donor kidney transplantation [45]. However, there is no evidence that eculizumab can be used for late AMR, and clinical trials are necessary to determine the optimal use of C5 inhibition.

3.5. Splenectomy. The spleen acts as a repository for memory $\mathrm{B}$ cells and plasma cells; thus, splenectomy is supposed to be effective in treating AMR. There is data suggesting that splenectomy alone can lead to rapid diuresis and immediate restoration of renal function [47]. Rescue splenectomy is currently regarded as last salvage option for AMR. There is a case that reported [60] that splenectomy is effective for treatment of CAMR after renal transplantation. However, clinical trials are needed to prove this finding.

\section{AMR in Liver Transplantation}

The liver allograft is generally regarded as relatively resistant to AMR. The resistance is attributed to a variety of characteristic features of liver which contribute to the clearing and dilution of antibodies or antigen-antibody complexes, such as Kupffer cell phagocytosis, large sinusoidal surface area, dual afferent hepatic blood supply, and secretion of soluble MHC class I antigen [61]. For many years, hyperacute rejection was thought not to occur, even when the $\mathrm{ABO}$ incompatible graft was used. However, subsequent increasing studies have shown that liver transplantation across the ABO blood type barrier $(\mathrm{ABO}$ ) is prone to $\mathrm{AMR}$, which often leads to a poor clinical outcome. Unlike a reliable tissue marker of AMR in renal and cardiac allografts, the diagnostic utility and functional significance of C4d immunostaining in the liver allograft are controversial and less clearly formed. There are reports that showed that extensive $\mathrm{C} 4 \mathrm{~d}$ deposition is associated with AMR and correlated with graft survival. However, C4d deposition in liver was also detected in several other conditions, such as acute cellular rejection, chronic rejection, and recurrent diseases including hepatitis $B$, hepatitis $C$, and autoimmune hepatitis, and even preservation injury [62-64]. Therefore, the diagnosis of AMR in liver cannot be made on the basis of histological finding alone and requires other supportive features as well as the exclusion of other causes of graft dysfunction that can mimic the pathological changes occurring in AMR. However, the presence of diffuse C4d immunostaining (involving endothelium or stroma in $>50 \%$ of portal tracts or sinusoids) provides supportive evidence for a diagnosis of AMR. Similar to renal transplantation, conventional T-cell-based immunosuppression usually seems less effective for cases with strictly defined AMR. Treatment with aggressive B-cell directed immunosuppression, including IVIG, plasmapheresis, and rituximab, is recommended to be 
used [61]. In animal models, antibody-mediated responses might play important roles in the development of chronic liver allograft rejection. However, the role of AMR in late liver graft loss is still underdetermined.

\section{AMR in Other Organ Transplantation}

AMR is also involved in other organ transplantation, especially for heart transplantation. DSA binding to the heart allograft causes myocardial injury predominantly through immune complex activation of the classical pathway of the complement cascade [65], and thus is a significant risk for allograft failure, cardiac allograft vasculopathy, and poor survival. C4d is accepted as a marker for AMR in cardiac allografts. The diagnosis of AMR has evolved from a clinical diagnosis to a primarily pathologic diagnosis based on histopathology and immunopathology. The ISHLT 2005 Working Formulation [66] recommended that AMR be diagnosed on the basis of (1) evaluation of the routinely processed and stained paraffin sections for endothelial-cell swelling and accumulations of intravascular macrophages; (2) immunophenotypic evidence of immunoglobulin (IgG, IgM, and/or IgA) and complement (C3d, C4d and/or Clq) deposition in capillaries by immunofluorescence (IF) on frozen sections and/or CD68 staining of intravascular macrophages in capillaries and C4d staining of capillaries by paraffin immunohistochemistry (IC). The final clinical diagnosis of AMR required evidence of allograft dysfunction and circulating donor-specific antibodies together with the histopathologic and immunophenotypic findings. Therapies include plasmapheresis, immunoadsorption columns, intravenous immune globulin, rituximab, and bortezomib. The combinations of steroid, IVIG, and plasmapheresis are suggested as initial therapies [67]. Late cardiac AMR caused by de novo DSA is also a serious problem; despite treatment consistent with current best practice, $46 \%$ of patients developed persistent cardiac dysfunction and their medium-term survival was poor [68].

Besides renal and heart transplantation, AMR is also a major complication causing graft injury after lung [69], pancreas [70], and intestinal [71] transplantation. Similarly, all the AMR are caused by DSA, and C4d is accepted as diagnosis marker. Antibody removal strategies are also used for these episodes. More studies are needed to understand these terms and improve their outcomes.

\section{Abbreviations}

AMR: Antibody-mediated rejection

CAMR: Chronic antibody-mediated rejection

DSA: Donor-specific antibody

HLA: Human lymphocyte antigen

IVIG: Intravenous immunoglobuin.

\section{Conflict of Interests}

The authors declared that they have no conflict of interests.

\section{Authors' Contribution}

Qiquan Sun and Yang Yang contributed equally to this work.

\section{Acknowledgments}

This study was supported by Grants from the General Program of National Natural Science Foundation of China (no. 81070593 and no. 81270834), a grant from Fund for Distinguished Young of Jiangsu Province, and a grant from 333 Talent Training Program of Jiangsu Province.

\section{References}

[1] R. A. Wolfe, V. B. Ashby, E. L. Milford et al., "Comparison of mortality in all patients on dialysis, patients on dialysis awaiting transplantation, and recipients of a first cadaveric transplant," New England Journal of Medicine, vol. 341, no. 23, pp. 1725-1730, 1999.

[2] C. Li and C. W. Yang, "The pathogenesis and treatment of chronic allograft nephropathy," Nature Reviews Nephrology, vol. 5, no. 9, pp. 513-519, 2009.

[3] B. J. Nankivell and D. R. Kuypers, "Diagnosis and prevention of chronic kidney allograft loss," The Lancet, vol. 378, no. 9800, pp. 1428-1437, 2011.

[4] R. B. Colvin and R. N. Smith, "Antibody-mediated organallograft rejection," Nature Reviews Immunology, vol. 5, no. 10, pp. 807-817, 2005.

[5] S. K. Takemoto, A. Zeevi, S. Feng et al., "National conference to assess antibody-mediated rejection in solid organ transplantation," American Journal of Transplantation, vol. 4, no. 7, pp. 1033-1041, 2004.

[6] G. Einecke, B. Sis, J. Reeve et al., "Antibody-mediated microcirculation injury is the major cause of late kidney transplant failure," American Journal of Transplantation, vol. 9, no. 11, pp. 2520-2531, 2009.

[7] J. Sellarés, D. G. De Freitas, M. Mengel et al., "Understanding the causes of kidney transplant failure: the dominant role of antibody-mediated rejection and nonadherence," American Journal of Transplantation, vol. 12, no. 2, pp. 388-399, 2012.

[8] R. B. Colvin, "Antibody-mediated renal allograft rejection: diagnosis and pathogenesis," Journal of the American Society of Nephrology, vol. 18, no. 4, pp. 1046-1056, 2007.

[9] L. C. Racusen, R. B. Colvin, K. Solez et al., "Antibody-mediated rejection criteria - an addition to the Banff 97 classification of renal allograft rejection," American Journal of Transplantation, vol. 3, no. 6, pp. 708-714, 2003.

[10] B. Sis, G. S. Jhangri, S. Bunnag, K. Allanach, B. Kaplan, and P. F. Halloran, "Endothelial gene expression in kidney transplants with alloantibody indicates Antibody-mediated damage despite lack of C4d staining," American Journal of Transplantation, vol. 9, no. 10, pp. 2312-2323, 2009.

[11] B. Sis and P. F. Halloran, "Endothelial transcripts uncover a previously unknown phenotype: $\mathrm{C} 4 \mathrm{~d}$-negative antibody-mediated rejection," Current Opinion in Organ Transplantation, vol. 15, no. 1, pp. 42-48, 2010.

[12] F. G. Cosio, J. M. Gloor, S. Sethi, and M. D. Stegall, “Transplant glomerulopathy," American Journal of Transplantation, vol. 8, no. 3, pp. 492-496, 2008.

[13] Q. Sun, X. Huang, S. Jiang, C. Zeng, and Z. Liu, "Picking transplant glomerulopathy out of the CAN: evidence from 
a clinico-pathological evaluation," BMC Nephrology, vol. 13, article 128, 2012.

[14] M. Haas, "Pathology of C4d-negative antibody-mediated rejection in renal allografts," Current Opinion in Organ Transplantation, vol. 18, pp. 319-326, 2013.

[15] P. F. Halloran and J. Sellares, "Microcirculation lesions alone are not reliable for identifying antibody-mediated rejection," American Journal of Transplantation, vol. 13, pp. 1931-1932, 2013.

[16] Q. Sun, D. Cheng, M. Zhang, Q. He, Z. Chen, and Z. Liu, "Predominance of intraglomerular T-bet or GATA3 may determine mechanism of transplant rejection," Journal of the American Society of Nephrology, vol. 22, no. 2, pp. 246-252, 2011.

[17] Q. Sun, M. Zhang, K. Xie et al., "Endothelial injury in transplant glomerulopathy is correlated with transcription factor T-bet expression," Kidney International, vol. 82, pp. 321-329, 2012.

[18] K. Solez, R. B. Colvin, L. C. Racusen et al., "Banff 07 classification of renal allograft pathology: updates and future directions," American Journal of Transplantation, vol. 8, no. 4, pp. 753-760, 2008.

[19] Q. Sun, Z.-H. Liu, S. Ji et al., "Late and early C4d-positive acute rejection: different clinico-histopathological subentities in renal transplantation," Kidney International, vol. 70, no. 2, pp. 377383, 2006.

[20] C. Dorje, K. Midtvedt, H. Holdaas et al., "Early versus late acute antibody-mediated rejection in renal transplant recipients," Transplantation, 2013.

[21] R. C. Walsh, P. Brailey, A. Girnita et al., "Early and late acute antibody-mediated rejection differ immunologically and in response to proteasome inhibition," Transplantation, vol. 91, no. 11, pp. 1218-1226, 2011.

[22] J. Pascual, M. D. Samaniego, J. R. Torrealba et al., "Antibodymediated rejection of the kidney after simultaneous pancreaskidney transplantation," Journal of the American Society of Nephrology, vol. 19, no. 4, pp. 812-824, 2008.

[23] J. R. Chapman, P. J. O’Connell, and B. J. Nankivell, "Chronic renal allograft dysfunction," Journal of the American Society of Nephrology, vol. 16, no. 10, pp. 3015-3026, 2005.

[24] K. Solez, R. B. Colvin, L. C. Racusen et al., "Banff 05 meeting report: differential diagnosis of chronic allograft injury and elimination of chronic allograft nephropathy (CAN)," American Journal of Transplantation, vol. 7, no. 3, pp. 518-526, 2007.

[25] P.-C. Lee, P. I. Terasaki, S. K. Takemoto et al., "All chronic rejection failures of kidney transplants were preceded by the development of HLA antibodies," Transplantation, vol. 74, no. 8, pp. 1192-1194, 2002.

[26] M. J. Everly, L. M. Rebellato, C. E. Haisch et al., "Incidence and impact of de novo donor-specific alloantibody in primary renal allografts," Transplantation, vol. 95, pp. 410-417, 2013.

[27] É. F. Campos, H. Tedesco-Silva, P. G. Machado, M. Franco, J. O. Medina-Pestana, and M. Gerbase-DeLima, "Post-transplant anti-HLA class II antibodies as risk factor for late kidney allograft failure," American Journal of Transplantation, vol. 6, no. 10, pp. 2316-2320, 2006.

[28] C. Wiebe, I. W. Gibson, T. D. Blydt-Hansen et al., "Evolution and clinical pathologic correlations of de novo donor-specific HLA antibody post kidney transplant," American Journal of Transplantation, vol. 12, no. 5, pp. 1157-1167, 2012.

[29] V. Nickeleit and M. J. Mihatsch, "Kidney transplants, antibodies and rejection: is C4d a magic marker?" Nephrology Dialysis Transplantation, vol. 18, no. 11, pp. 2232-2239, 2003.
[30] N. Singh, Q. Sun, T. Nadasdy et al., "The pathogenesis of acute allograft dysfunction in desensitized renal transplant recipients," Clinical Transplantation, vol. 26, pp. E402-E411, 2012.

[31] C. Lefaucheur, A. Loupy, D. Vernerey et al., "Antibody-mediated vascular rejection of kidney allografts: a population-based study," The Lancet, vol. 381, pp. 313-319, 2013.

[32] J. A. Bradley, W. M. Baldwin, A. Bingaman et al., "Antibodymediated rejection-an ounce of prevention is worth a pound of cure," American Journal of Transplantation, vol. 11, no. 6, pp. 1131-1139, 2011.

[33] C. Morath, G. Opelz, M. Zeier, and C. Susal, "Prevention of antibody-mediated kidney transplant rejection," Transplant International, vol. 25, pp. 633-645, 2012.

[34] T. P. Theruvath, S. L. Saidman, S. Mauiyyedi et al., "Control of antidonor antibody production with tacrolimus and mycophenolate mofetil in renal allograft recipients with chronic rejection," Transplantation, vol. 72, no. 1, pp. 77-83, 2001.

[35] Q. Sun, Z.-H. Liu, Z. Cheng et al., “Treatment of early mixed cellular and humoral renal allograft rejection with tacrolimus and mycophenolate mofetil," Kidney International, vol. 71, no. 1, pp. 24-30, 2007.

[36] Q. Sun, Z.-H. Liu, G. Yin et al., "Tacrolimus combined with mycophenolate mofetil can effectively reverse C4d-positive steroid-resistant acute rejection in Chinese renal allograft recipients," Nephrology Dialysis Transplantation, vol. 21, no. 2, pp. 510-517, 2006.

[37] M. Pascual, S. Saidman, N. Tolkoff-Rubin et al., "Plasma exchange and tacrolimus-mycophenolate rescue for acute humoral rejection in kidney transplantation," Transplantation, vol. 66, pp. 1460-1464, 1999.

[38] G. A. Böhmig, H. Regele, M. Exner et al., "C4d-positive acute humoral renal allograft rejection: effective treatment by immunoadsorption," Journal of the American Society of Nephrology, vol. 12, no. 11, pp. 2482-2489, 2001.

[39] S. C. Jordan, A. W. Quartel, L. S. C. Czer et al., "Posttransplant therapy using high-dose human immunoglobulin (intravenous gammaglobulin) to control acute humoral reaction in renal and cardiac allograft recipients and potential mechanism of action," Transplantation, vol. 66, no. 6, pp. 800-808, 1998.

[40] D. H. Casadei, M. Del C. Rial, G. Opelz et al., "A randomized and prospective study comparing treatment with high-dose intravenous immunoglobulin with monoclonal antibodies for rescue of kidney grafts with steroid-resistant rejection," Transplantation, vol. 71, no. 1, pp. 53-58, 2001.

[41] S. Faguer, N. Kamar, C. Guilbeaud-Frugier et al., "Rituximab therapy for acute humoral rejection after kidney transplantation," Transplantation, vol. 83, no. 9, pp. 1277-1280, 2007.

[42] W. R. Mulley, F. J. Hudson, B. D. Tait et al., "A single low-fixed dose of rituximab to salvage renal transplants from refractory antibody-mediated rejection," Transplantation, vol. 87, no. 2, pp. 286-289, 2009.

[43] M. J. Everly, J. J. Everly, B. Susskind et al., "Bortezomib provides effective therapy for antibody- and cell-mediated acute rejection," Transplantation, vol. 86, no. 12, pp. 1754-1761, 2008.

[44] S. M. Flechner, R. Fatica, M. Askar et al., "The role of proteasome inhibition with bortezomib in the treatment of antibodymediated rejection after kidney-only or kidney-combined organ transplantation," Transplantation, vol. 90, no. 12, pp. 1486-1492, 2010. 
[45] F. Gonzalez-Roncero, M. Suner, G. Bernal et al., "Eculizumab treatment of acute antibody-mediated rejection in renal transplantation: case reports," Transplantation Proceedings, vol. 44, pp. 2690-2694, 2012.

[46] J. E. Locke, C. M. Magro, A. L. Singer et al., "The use of antibody to complement protein $\mathrm{C} 5$ for salvage treatment of severe antibody-mediated rejection," American Journal of Transplantation, vol. 9, no. 1, pp. 231-235, 2009.

[47] J. E. Locke, A. A. Zachary, M. Haas et al., "The utility of splenectomy as rescue treatment for severe acute antibody mediated rejection," American Journal of Transplantation, vol. 7, no. 4, pp. 842-846, 2007.

[48] B. Kaplan, A. Gangemi, J. Thielke, J. Oberholzer, H. Sankary, and E. Benedetti, "Successful rescue of refractory, severe antibody mediated rejection with splenectomy," Transplantation, vol. 83, no. 1, pp. 99-100, 2007.

[49] H. Billing, S. Rieger, J. Ovens et al., "Successful treatment of chronic antibody-mediated rejection with IVIG and rituximab in pediatric renal transplant recipients," Transplantation, vol. 86, no. 9, pp. 1214-1221, 2008.

[50] T. Fehr, B. Rüsi, A. Fischer, H. Hopfer, R. P. Wüthrich, and A. Gaspert, "Rituximab and intravenous immunoglobulin treatment of chronic antibody-mediated kidney allograft rejection," Transplantation, vol. 87, no. 12, pp. 1837-1841, 2009.

[51] Y. A. Hong, H. G. Kim, S. R. Choi et al., "Effectiveness of rituximab and intravenous immunoglobulin therapy in renal transplant recipients with chronic active antibody-mediated rejection," Transplantation Proceedings, vol. 44, no. 1, pp. 182184, 2012.

[52] R. N. Smith, F. Malik, and N. Goes, "Partial therapeutic response to Rituximab for the treatment of chronic alloantibody mediated rejection of kidney allografts," Transplant Immunology, vol. 27, pp. 107-113, 2012.

[53] D. K. Perry, J. M. Burns, H. S. Pollinger et al., "Proteasome inhibition causes apoptosis of normal human plasma cells preventing alloantibody production," American Journal of Transplantation, vol. 9, no. 1, pp. 201-209, 2009.

[54] R. C. Walsh, R. R. Alloway, A. L. Girnita, and E. S. Woodle, "Proteasome inhibitor-based therapy for antibody-mediated rejection," Kidney International, vol. 81, pp. 1067-1074, 2012.

[55] E. S. Woodle and M. Rubin, "Proteasome inhibitor therapy for antibody mediated rejection: initial report from a multicenter collaborative," American Journal of Transplantation, vol. 10, no. 4: article 83, 2010.

[56] J. Ashton-Chess, H. L. Mai, V. Jovanovic et al., "Immunoproteasome beta subunit 10 is increased in chronic antibody-mediated rejection," Kidney International, vol. 77, no. 10, pp. 880-890, 2010.

[57] N. Alachkar, S. M. Bagnasco, and R. A. Montgomery, "Eculizumab for the treatment of two recurrences of atypical hemolytic uremic syndrome in a kidney allograft," Transplant International, vol. 25, pp. e93-e95, 2012.

[58] M. D. Stegall, T. Diwan, S. Raghavaiah et al., "Terminal complement inhibition decreases antibody-mediated rejection in sensitized renal transplant recipients," American Journal of Transplantation, vol. 11, no. 11, pp. 2405-2413, 2011.

[59] Z. A. Stewart, T. E. Collins, A. J. Schlueter et al., "Case report: Eculizumab rescue of severe accelerated antibody-mediated rejection after $\mathrm{ABO}$-incompatible kidney transplant," Transplantation Proceedings, vol. 44, pp. 3033-3036, 2012.

[60] H. Ishida, K. Omoto, T. Shimizu et al., "Usefulness of splenectomy for chronic active antibody-mediated rejection after renal transplantation," Transplant International, vol. 21, no. 6, pp. 602-604, 2008.

[61] S. G. Hubscher, "Antibody-mediated rejection in the liver allograft," Current Opinion in Organ Transplantation, vol. 17, pp. 280-286, 2012.

[62] C. O. C. Bellamy, "Complement C4d immunohistochemistry in the assessment of liver allograft biopsy samples: applications and pitfalls," Liver Transplantation, vol. 17, no. 7, pp. 747-750, 2011.

[63] S. Ali, A. Ormsby, V. Shah et al., "Significance of complement split product $\mathrm{C} 4 \mathrm{~d}$ in $\mathrm{ABO}-$ compatible liver allograft: diagnosing utility in acute antibody mediated rejection," Transplant Immunology, vol. 26, no. 1, pp. 62-69, 2012.

[64] U. P. Neuman and P. Neuhaus, "C4d immunostaining in acute humoral rejection after $\mathrm{ABO}$ blood group-incompatible liver transplantation," Liver Transplantation, vol. 12, no. 3, pp. 356357, 2006.

[65] K. Murata and W. M. Baldwin III, "Mechanisms of complement activation, C4d deposition, and their contribution to the pathogenesis of antibody-mediated rejection," Transplantation Reviews, vol. 23, no. 3, pp. 139-150, 2009.

[66] S. Stewart, G. L. Winters, M. C. Fishbein et al., "Revision of the 1990 working formulation for the standardization of nomenclature in the diagnosis of heart rejection," Journal of Heart and Lung Transplantation, vol. 24, no. 11, pp. 1710-1720, 2005.

[67] S. Chih, K. J. Tinckam, and H. J. Ross, "A survey of current practice for antibody-mediated rejection in heart transplantation," American Journal of Transplantation, vol. 13, pp. 10691074, 2013.

[68] A. M. Hodges, H. Lyster, A. McDermott et al., "Late antibodymediated rejection after heart transplantation following the development of de Novo Donor-specific human leukocyte antigen antibody," Transplantation, vol. 93, no. 6, pp. 650-656, 2012.

[69] R. Hachem, "Antibody-mediated lung transplant rejection," Current Respiratory Care Reports, vol. 1, pp. 157-161, 2012.

[70] C. B. Drachenberg, J. R. Torrealba, B. J. Nankivell et al., "Guidelines for the diagnosis of antibody-mediated rejection in pancreas allografts-updated Banff grading schema," American Journal of Transplantation, vol. 11, no. 9, pp. 1792-1802, 2011.

[71] A. A. S. Dick and S. Horslen, "Antibody-mediated rejection after intestinal transplantation," Current Opinion in Organ Transplantation, vol. 17, pp. 250-257, 2012. 


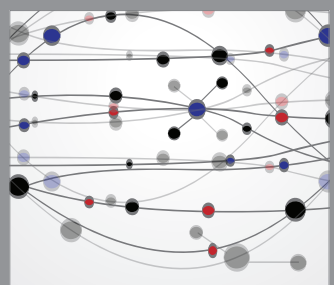

The Scientific World Journal
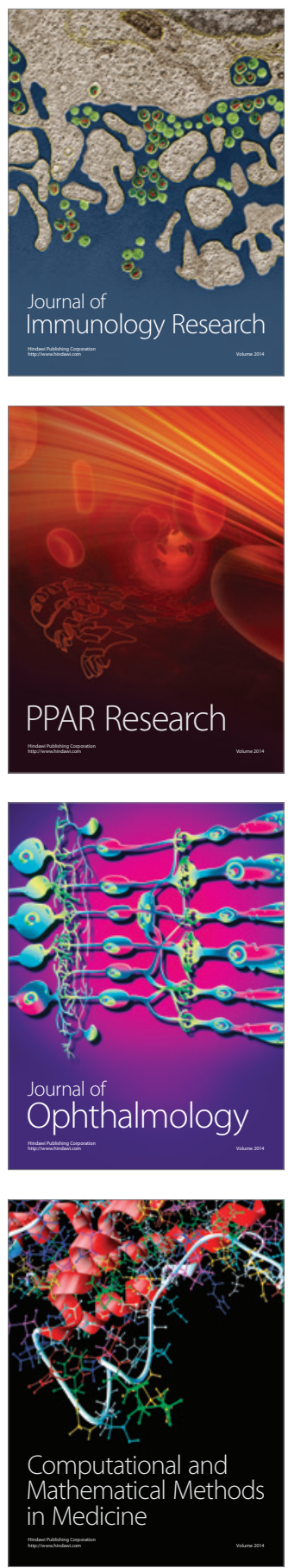

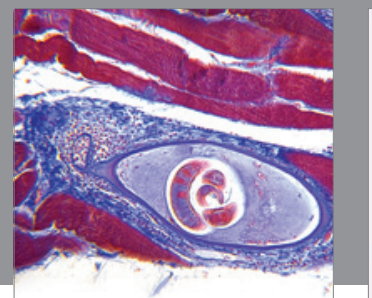

Gastroenterology

Research and Practice
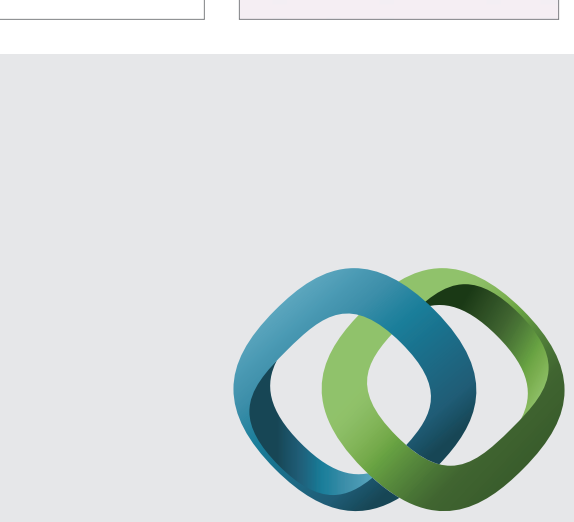

\section{Hindawi}

Submit your manuscripts at

http://www.hindawi.com
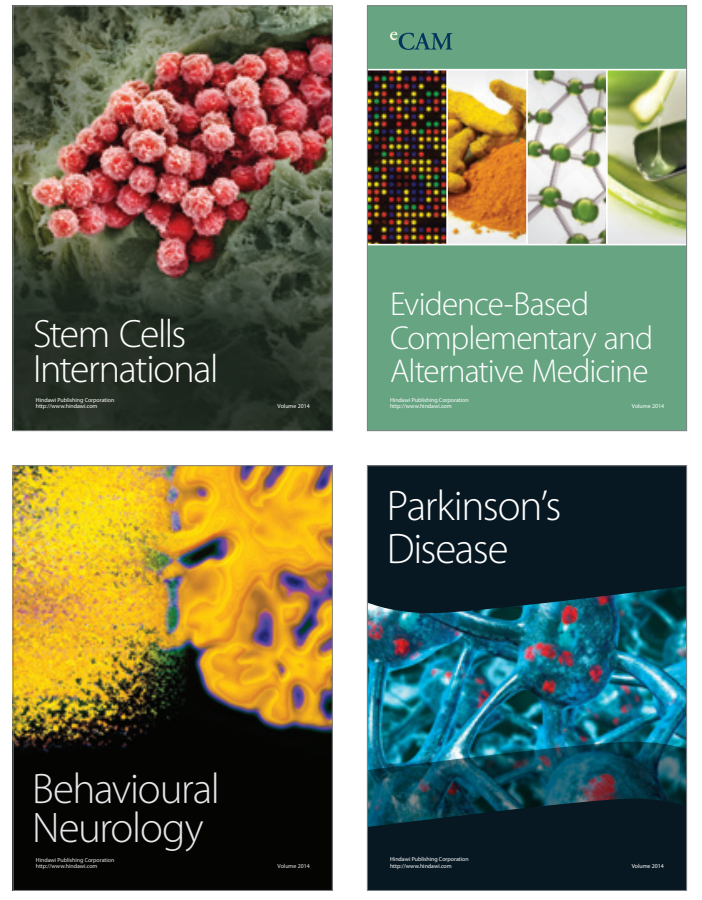
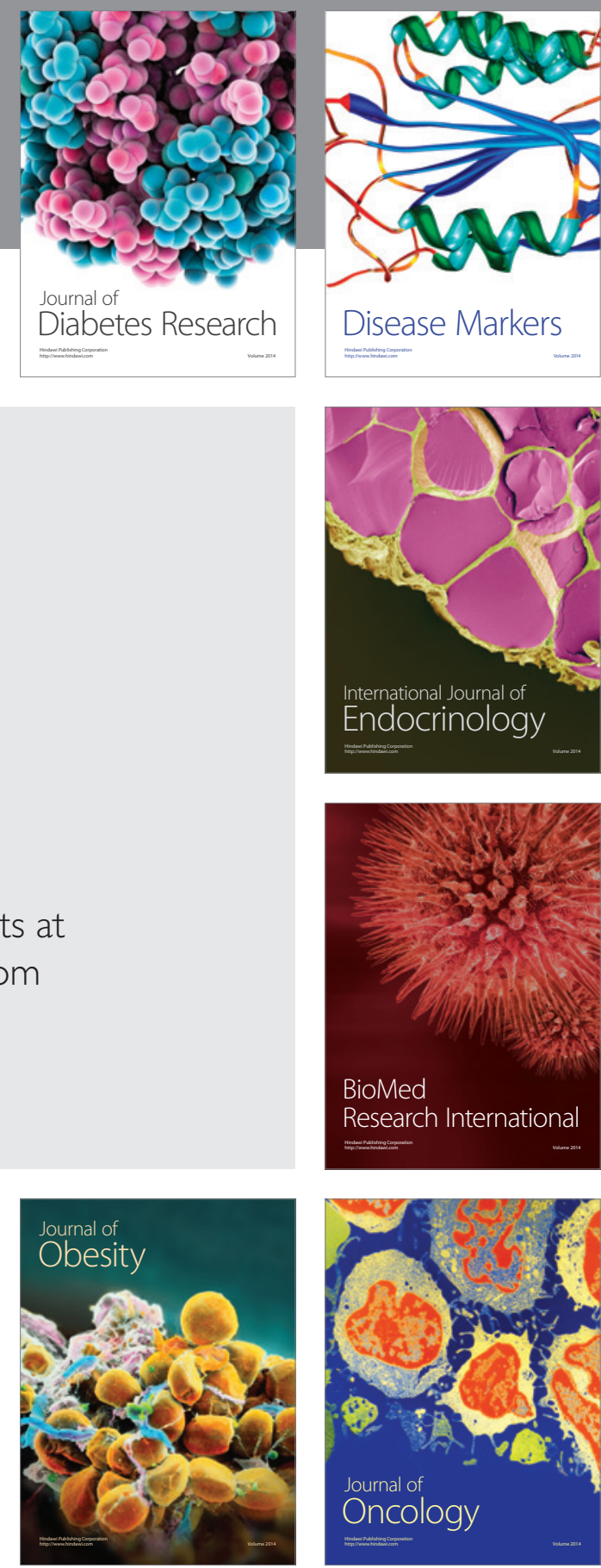

Disease Markers
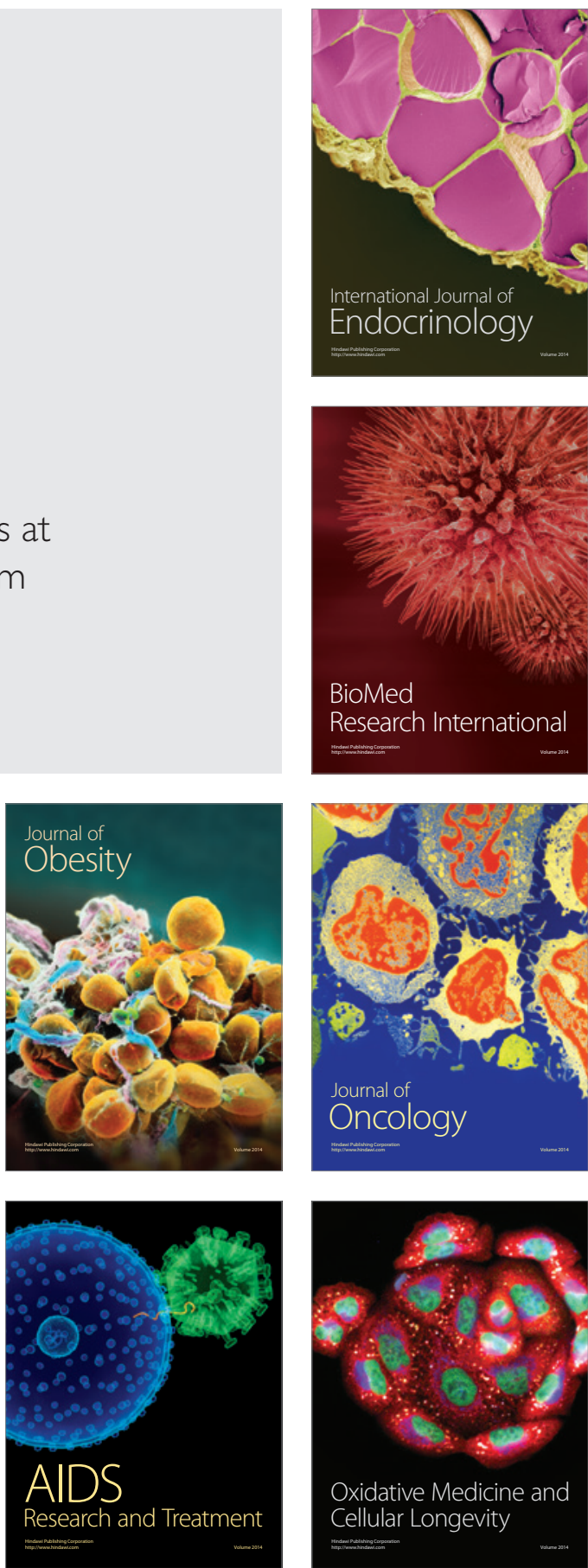